\title{
Influence of the theoretical load point on the value of the $J$ - JQ integral during determination of fracture toughness of 2519 aluminium alloy
}

\author{
Maciej $\mathrm{Kotyk}^{1,}{ }^{*}$, and Dariusz Boroński ${ }^{1}$ \\ ${ }^{1}$ University of Science and Technology, Faculty of Mechanical Engineering, Al. prof. S. Kaliskiego 7, \\ 85-796 Bydgoszcz
}

\begin{abstract}
The J integral is a value describing the fracture toughness of nonlinear-elastic materials. One of such materials is the aluminum alloy AA2519. Due to the fact that it became the base material for the composite AA2519 - AA1050 - Ti6A14V, it was decided to re-test this material. Due to the use of the aforementioned Al - Ti composite alloy AA2519 was also tested under cryogenic conditions. During the tests, its fracture toughness was determined using the $\mathrm{J}$ integral. Both in the method of one sample and many samples it is necessary to include the geometry of the sample. Currently, standard methods withdrawn, and did not describe all the required aspects of the research. During the time when the described material was created, the displacement point was not taken into account during the fracture toughness tests. Accordingly, there is a need to re-verify the determined once the fracture toughness of the described material. This article presents the author's method of determining load displacement lines in compact samples (CT). It should be noted that this sample had an uncommonly encountered geometry. In the described case the width of the sample was four times the thickness.
\end{abstract}

\section{Introduction}

Construction materials improve their properties. This is mainly due to market requirements. Naturally, technological progress is the reason for this. This development can be seen in the aerospace industry as well as in military applications. This is not limited only to the design of new machines or devices. The described progress can also be observed by creating new materials, or by finding a new application for already existing materials, which are widely used and described in the literature. Sometimes such a material can also become one of the elements of the composite and in combination with other material can obtain new, interesting for its potential application properties. An example of such a group of materials are aluminum alloys. Due to the possibility of applying the anode, resistance to corrosion and much lower density than for example steel, the material described increases its popularity and the number of applications. To give the surface composite made of aluminum alloys special properties, these alloys are combined with other materials. It is possible to do this using various

*Corresponding author: maciej.kotyk@utp.edu.pl 
technologies. They are, for example, welding, soldering, friction welding and high energy methods such as explosive welding [1-2].

The consequence of the creation of such materials is to have knowledge about their mechanical characteristics. Not without significance is also the knowledge of the properties of the base materials from which the composite is produced. This information can be used to develop models that allow to determine the material properties of layered materials based on the properties of the constituent materials.

Determination of mechanical properties for layered materials causes many ambiguities. Although the characteristics of the tensile test are not subject to high ambiguity. However, in the case of measuring volumes during which the load is cyclical, the results of the tests are difficult to interpret. This is because there are at least two different materials in the composite, often with significantly different mechanical properties. Interaction takes place between the materials. This connection is completely different than, for example adhesive joints [3-4]

An example of such scientific research is the determination of fatigue life and fracture mechanics. This is because the precrack is generated by cyclic load. A good example of this is research to determine the $\mathrm{J}$ integral. One of the layered materials is an explosion-welded plater composed of three materials AA2519 - AA1050 - Ti6A14V. Its intended application is to use it in aerospace and space constructions [5]. Due to its innovativeness, it is necessary to know its mechanical characteristics, including fracture toughness.

As mentioned before, to develop a method for describing the fracture toughness of a laminar material, is to know the mechanical properties of the base materials. Due to this, it was decided to determine the experimental value of $\mathrm{J}-\mathrm{J}_{\mathrm{Q}}$ integral for aluminum alloy AA2519.

\section{Test conditions}

The reference point for fracture toughness tests is the Al - Ti composite geometry. Due to the only available sheet thickness of the laminar material which was $10 \mathrm{~mm}$, samples for testing of base materials, were also decided to perform with described thickness. It should be noted that the geometry of the sample was developed in accordance with recommendations of standard. The dimensions of the sample are presented in Figure 1.

a)

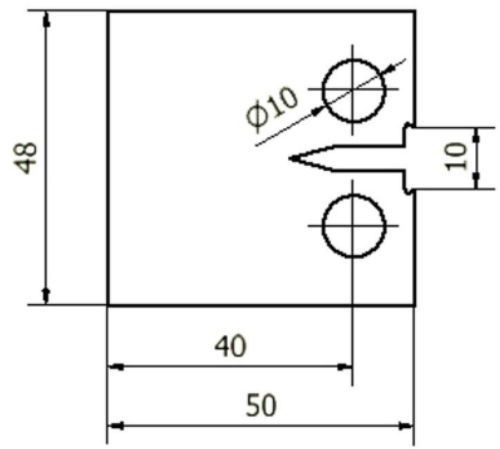

b)

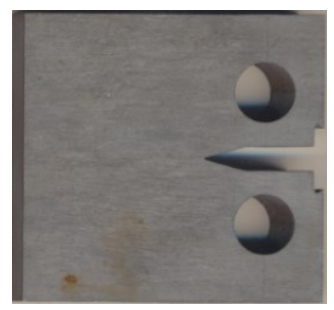

Fig. 1. A sample for fracture toughness tests, a) basic dimensions of the sample, b) a real sample.

Samples were tested using a standard Instron 8501 testing machine with a hydraulic power supply system. Before the start of the main part of the research, a precrack was generated in the samples. Automation of fatigue cracks was ensured by a system of cameras placed on both sides of the sample. The images recorded by the cameras were analyzed using algorithms based on digital image correlation. The software calculates the displacement 
gradient around the apex of the crack so that the location of the crack tip was known. After reaching the critical value of the user-defined crack length, the testing machine was stopped. The research stand with digital cameras mounted on it is presented in Figure 2. The method of operation software was presented in figure 3. Detailed information about the system operation can be found in the article [6]. It should be noted that the crack opening displacement was carried out by placing a CT sample extensometer in the lock, as shown in figure 2 .

a)

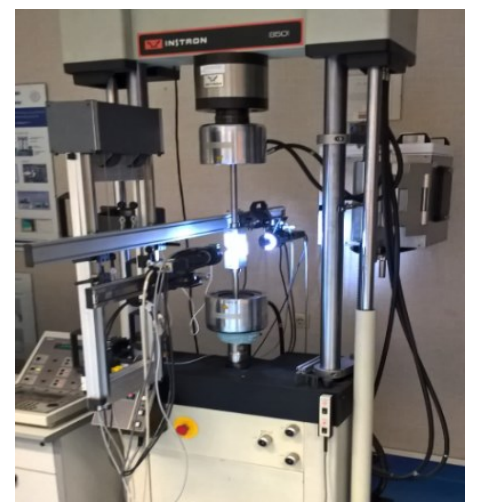

b)

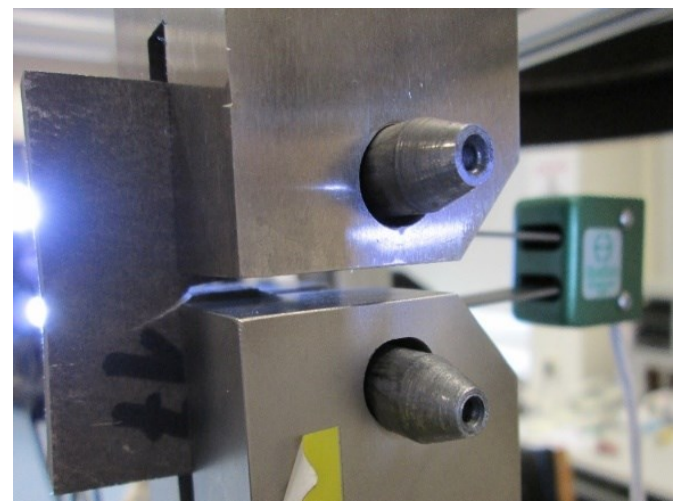

Fig. 2. Test stand, a) fatigue crack generation, b) measurement of crack opening displacement.

a)

b)

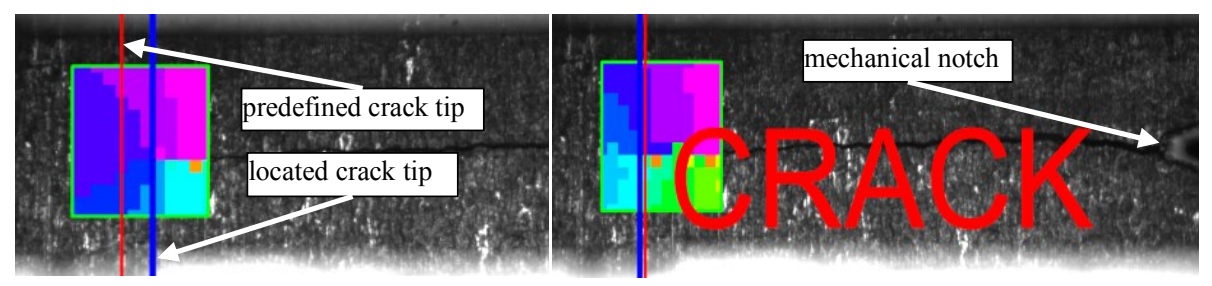

Fig. 3. Detecting the crack tip, a) crack tip in front of the boundary line, b) crack top after the boundary line.

According to the test procedure defined in the American standard [7], the displacement of the point of application of force should be measured in its axis. Due to the previously presented sample geometry, it was not possible to mount the extensometer directly at the load line. Therefore, it was decided to convert the results obtained taking into account the geometry of the sample. To do this, model was prepared which is shown in Figure 4. 


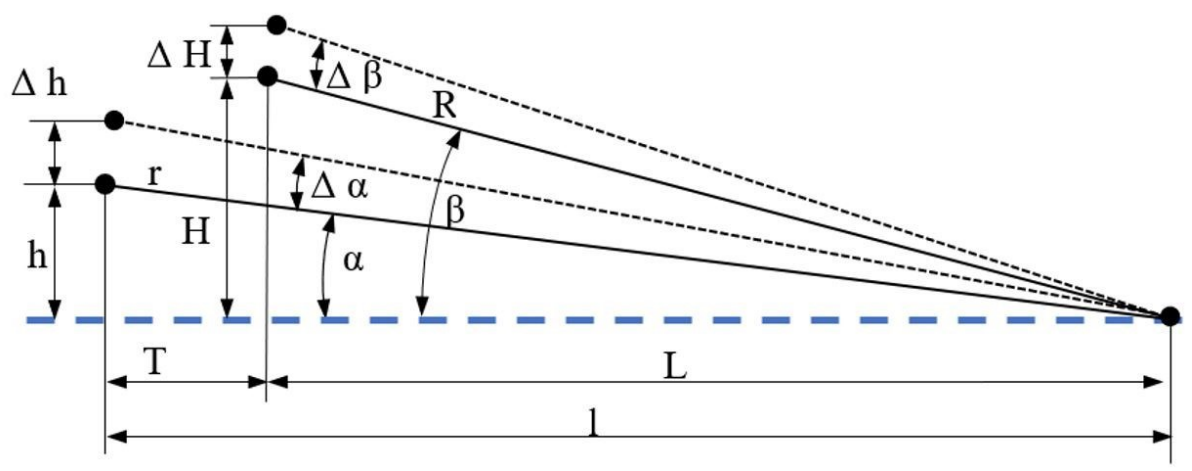

Fig. 4. Graphical representation of the geometrical relationships that were taken to calculate the displacement of the measurement point to the displacement at the load line.

The illustration shows that to calculate the opening of the sample measured at its edge, to the point of displacement of the force line, you should use the following relationships:

$$
\begin{gathered}
\frac{H+\Delta H}{R}=\sin (\beta+\Delta \alpha) \\
\frac{h+\Delta h}{r}=\sin (\alpha+\Delta \alpha) \\
\alpha+\Delta \alpha=\arcsin \left(\frac{h+\Delta h}{r}\right) \\
\Delta \alpha=\arcsin \left(\frac{h+\Delta h}{r}\right)-\alpha
\end{gathered}
$$

and finally:

$$
\Delta H=R \cdot \sin \left(\beta+\arcsin \left(\frac{h+\Delta h}{r}\right)-\alpha\right)-H
$$

where:

$$
\begin{aligned}
& l=35,25 \mathrm{~mm} \\
& H=11 \mathrm{~mm} \\
& B=10 \mathrm{~mm} \\
& L=25,25 \mathrm{~mm}
\end{aligned}
$$

and:

$$
\begin{gathered}
L=\left(\frac{a+W}{2}\right) \\
r=\sqrt{h^{2}+(T+L)^{2}} \\
R=\sqrt{H^{2}+(L)^{2}} \\
\frac{h}{T+L}=\operatorname{tg}(\alpha) \rightarrow \alpha \\
\frac{H}{L}=\operatorname{tg}(\beta) \rightarrow \beta
\end{gathered}
$$

Above relationships are true if:

$$
\Delta \alpha=\Delta \beta
$$

After applying the presented mathematical model, the measurement points were considered as a displacement operating in the load line. 


\section{Experiment procedure}

The tests were carried out in accordance with the test procedure described in the relevant international standard. The sample with the previously generated fatigue crack was loaded cyclically. It should be noted that the handles were made so that a treadmill was formed in the mounting holes, on which the pins could roll. As a result, the frictional moment between the bolts and the sample is kept to a minimum. The testing machine was controlled by a displacement channel. After reaching user-specified displacement, the unloading was $15 \%$ of the force. After this, the sample was loaded again to obtain the next displacement of the machine piston. The procedure was repeated until the sample was destroyed. As a result, we obtained graphs of load - crack opening displacement, which were calculated in accordance with the relationships presented in the previous chapter.

\section{Sample results and discussion}

Due to the discrepancies that may arise from the measurement of the crack opening in the sample lock and not in the force line, it was decided to compare the results from before and after the correction. To determine the size of the described differences, the $J$ integral waveforms were compared with the crack length increment, not corrected with the $\mathrm{J}$ integral graph the crack length increase, but after correction. To determine the size of the described differences, the individual values of the integral $J$ were compared. Some of them were corrected, while the other was not. The results presented are a selected fragment from the fracture toughness tests of the previously discussed AA2519 aluminum alloy tested under room conditions. Thus, the graph showing the important measuring points after the correction and the corresponding points, but not corrected, are shown in Figure 5.

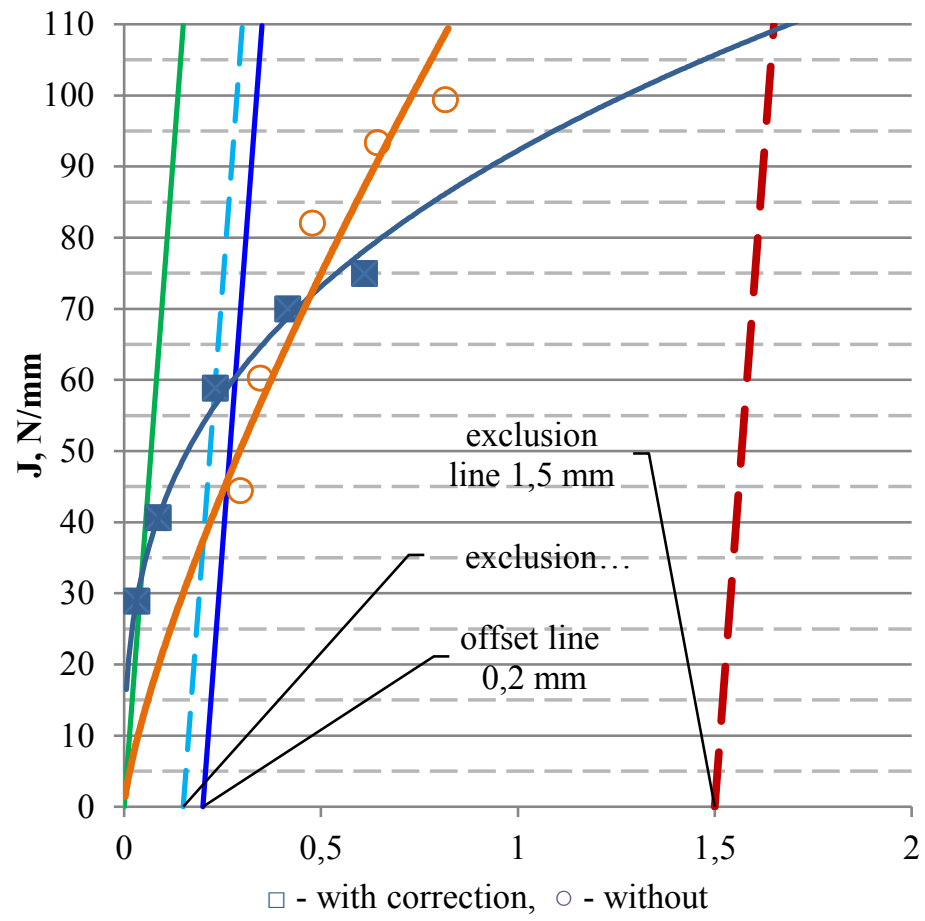

Fig.5. A comparison of the partial values of the J integral for AA2519 determined with and without the force displacement point correction. 
From the graph above, it can be clearly seen that the introduction of the correction significantly influences the value of the partial values of the integral J. The introduction of the correction resulted in shifting all points in the direction of the abscissae. As a result, the number of measuring points has decreased, which from the point of view of the test procedure are considered important. In addition, the offset of points as a result of the correction ultimately influenced its value. Apart from the minimum number of points necessary to determine the experimental $\mathrm{J}-\mathrm{J}_{\mathrm{Q}}$ integral that correction introduced a decrease in the characteristic value for fracture toughness in the elastic - plastic range. In the analyzed case, it decreased from $60 \mathrm{~N} / \mathrm{mm}$ to $47 \mathrm{~N} / \mathrm{mm}$, which is a $22 \%$ decrease. Not taking into account the rotation of the sample caused a decrease in the model's fit coefficient for the power curve characteristic for the J-integral analysis by one sample method. For results without correction, it was 0.9 , while after its performance this value reached 0.99 .

\section{Conclusions}

Based on the tests carried out and the analysis of their results, conclusions were drawn

- The introduction of the load point displacement correction in the analyzed case significantly influences the value of the individual values of $\mathrm{J}$ integral.

- Correction also changes the very nature of the approximation line. As a result, it was possible to describe the approximation curve with the value of parameter $\mathrm{R}^{2}$ almost equal to 1 .

- After the correction, the number of points in the area between the exclusion lines was smaller than the minimum value determined by the normative determinants to determine of $\mathrm{J}$ integral. This causes difficulties in determining the critical value that can be considered as fracture toughness.

\section{References}

1 D. Kocańda, A. Górka, Biul. Wojsk. Akad. Tech., 59, 395-411 (2010)

2 D. Boroński, M. Kotyk, P. Maćkowiak, L. Śnieżek, Mater. Des., 133, 390-403 (2017)

3 P. Maćkowiak, B. Ligaj, Eng. Mech., pp. 598-601 (2017)

4 P. Maćkowiak, D. Płaczek, M. Kotyk, IOP Conf. Ser. Mater. Sci. Eng., 393, 012-027 (2018)

5 I. Szachogluchowicz, L. Sniezek, V. Hutsaylyuk, Eng. Fail. Anal., 69, 77-87 (2016)

6 R. Sołtysiak, D. Boroński, M. Kotyk, Am. Inst. Phys., 050006 (2016)

7 ASTM E1820-18, "Standard Test Method for Measurement of Fracture Toughness," ASTM Int. (2018) 